\title{
Bilinear isometries on subspaces of continuous functions
}

\author{
Juan J. Font ${ }^{* 1}$ and M. Sanchis** 1 \\ ${ }^{1}$ Departament de Matemàtiques, Universitat Jaume I, Campus del Riu Sec, 12071 Castelló, Spain
}

Received 14 December 2006, revised 24 September 2008, accepted 5 October 2008

Published online 18 March 2010

Key words Bilinear isometry, subspaces of continuous functions, generalized peak point MSC (2000) 46A55, 46E15

Let $A$ and $B$ be strongly separating linear subspaces of $C_{0}(X)$ and $C_{0}(Y)$, respectively, and assume that $\partial A \neq$ $\emptyset\left(\partial A\right.$ stands for the set of generalized peak points for $A$ ) and $\partial B \neq \emptyset$. Let $T: A \times B \longrightarrow C_{0}(Z)$ be a bilinear isometry. Then there exist a nonempty subset $Z_{0}$ of $Z$, a surjective continuous mapping $h: Z_{0} \longrightarrow \partial A \times \partial B$ and a norm-one continuous function $a: Z_{0} \longrightarrow K$ such that $T(f, g)(z)=a(z) f\left(\pi_{x}(h(z)) g\left(\pi_{y}(h(z))\right.\right.$ for all $z \in Z_{0}$ and every pair $(f, g) \in A \times B$. These results can be applied, for example, to non-unital function algebras.

(C) 2010 WILEY-VCH Verlag GmbH \& Co. KGaA, Weinheim

\section{Introduction}

Let $X$ be a locally compact Hausdorff space. As usual, $C_{0}(X)$ (resp. $C(X)$ if $X$ is compact) stands for the Banach space of all continuous scalar-valued functions on $X$ which vanish at infinity, endowed with the supremum norm, $\|\cdot\|_{\infty}$. In [6], the authors proved the following bilinear version of the well-known Holsztyński's Theorem on non-surjective linear isometries of $C(X)$-spaces ([5]):

Let $T: C(X) \times C(Y) \longrightarrow C(Z)$ be a bilinear isometry. Then there exist a closed subset $Z_{0}$ of $Z$, a surjective continuous mapping $h: Z_{0} \longrightarrow X \times Y$ and a norm-one continuous function $a \in C(Z)$ such that $T(f, g)(z)=a(z) f\left(\pi_{x}(h(z)) g\left(\pi_{y}(h(z))\right.\right.$ for all $z \in Z_{0}$ and every pair $(f, g) \in C(X) \times C(Y)$.

The proof of this result rests heavily on the powerful Stone-Weierstrass Theorem. In this paper we extend this bilinear version of Holsztyński's Theorem to a more general context, where Stone-Weierstrass Theorem is not applicable (see Theorem 3.6). Our version is valid, among others, for completely regular (in particular, extremely regular) subspaces of $C_{0}(X)$ and for non-unital function algebras.

\section{Preliminaries}

Let $X$ be a locally compact space and $A$ be a linear subspace of $C_{0}(X)$. It is said that $A$ is separating (resp. strongly separating ([1])) if for distinct $x, y \in X$, there exists $f \in A$ such that $f(x) \neq f(y)$ (resp. $|f(x)| \neq$ $|f(y)|)$.

Let $x_{0} \in X$. It is said that $x_{0}$ is a generalized peak point (also called strong boundary point or weak peak point) for $A$ if for every open neighborhood, $V$, of $x_{0}$ there exists $f \in A$ such that $\|f\|=\left|f\left(x_{0}\right)\right|=1$ and $f$ vanishes outside $V$. We shall write $\partial A$ to denote the set of generalized peak points for $A$ and $C h(A)$ to denote the Choquet boundary for $A$, which is to say, the subspace of $X$ consisting of the extreme points of the closed unit ball of the dual of $A$.

* e-mail: font@mat.uji.es

** Corresponding author: e-mail: sanchis@mat.uji.es 


\section{Bilinear isometries}

In the sequel, we shall assume that $A$ and $B$ are nonvoid linear subspaces of $C_{0}(X)$ and $C_{0}(Y)$, respectively, whose sets of generalized peak points are not empty (see Remark 3.7 below). Furthermore, $T: A \times B \longrightarrow C_{0}(Z)$ will be a bilinear mapping which satisfies

$$
\|T(f, g)\|=\|f\|\|g\|
$$

for every $(f, g) \in A \times B$, which is to say that $T$ is a bilinear isometry.

For any $x \in X$, let

$$
C_{x}:=\{f \in A: 1=\|f\|=|f(x)|\} .
$$

Lemma 3.1 Assume $(x, y) \in \partial A \times \partial B$. The set

$$
I_{x, y}:=\left\{z \in Z: 1=\|T(f, g)\|=\mid\left(T(f, g)(z) \mid \text { for }(f, g) \in C_{x} \times C_{y}\right\}\right.
$$

is nonempty.

Proof. For any $f \in A$ and $g \in B$, let $L(f, g):=\{z \in Z:\|T(f, g)\|=|T(f, g)(z)|\}$ and let $M_{f, g}:=$ $\left\{z \in Z:|T(f, g)(z)| \geq \frac{\|T(f, g)\|}{2}\right\}$ which is compact since $T(f, g) \in C_{0}(Z)$. To prove that $I_{x, y}$ is nonempty, and since $I_{x, y}$ is a closed subset of $M_{f, g}$, we shall check that if $f_{1}, \ldots, f_{n}$ belong to $C_{x}$ and $g_{1}, \ldots, g_{n}$ belong to $C_{y}$, then $\bigcap_{i, j} L\left(f_{i}, g_{j}\right) \neq \emptyset$. Let $f \in A$ and $g \in B$ defined as follows:

$$
f:=\sum_{i=1}^{n} \frac{\left|f_{i}(x)\right|}{f_{i}(x)} f_{i}
$$

and

$$
g:=\sum_{j=1}^{n} \frac{\left|g_{j}(y)\right|}{g_{i}(y)} g_{i}
$$

It is clear that $|f(x)|=n=\|f\|$ and $|g(y)|=n=\|g\|$. Hence, $\|T(f, g)\|=\|f\|\|g\|=n^{2}$ since $T$ is a bilinear isometry and there exists $z \in Z$ such that

$$
|T(f, g)(z)|=n^{2}=\left|\sum_{i, j} \frac{\left|f_{i}(x)\right|}{f_{i}(x)} \frac{\left|g_{j}(y)\right|}{g_{j}(y)} T\left(f_{i}, g_{j}\right)(z)\right| .
$$

As $\left\|T\left(f_{i}, g_{j}\right)\right\| \leq 1$ for every $i, j$, we infer that $\left|T\left(f_{i}, g_{j}\right)(z)\right|=1$, which is to say that $z \in \bigcap_{i, j} L\left(f_{i}, g_{j}\right)$, as was to be proved.

Lemma 3.2 Assume $\left(x_{0}, y\right) \in \partial A \times \partial B$. Fix $g \in C_{y}$ and define a linear isometry $S: A \longrightarrow C_{0}(Z)$ as $S(f):=T(f, g)$. If $f\left(x_{0}\right)=0$, then $(S f)(z)=0$ for all $z \in I_{x_{0}, y}$.

Proof. Assume there exists $z_{0} \in I_{x_{0}, y}$ such that $(S f)\left(z_{0}\right) \neq 0$ for some $f \in A$. Let us assume that $\|f\|=1$ and $(S f)\left(z_{0}\right)=\alpha$ with $0<\alpha \leq 1$. Let $U=\left\{x \in X:|f(x)| \geq \frac{\alpha}{2}\right\}$. There is $f^{\prime} \in A$ such that $1=\left\|f^{\prime}\right\|=\left|f^{\prime}\left(x_{0}\right)\right|,\left|f^{\prime}(x)\right|<1$ for all $x \in U$ and, multiplying by a constant if necessary, $\left(S f^{\prime}\right)\left(z_{0}\right)=1$. Since $U$ is compact, there exists $s:=\sup _{x \in U}\left\{\left|f^{\prime}(x)\right|\right\}<1$. Then we can find a positive integer $M$ such that $1+M s<\alpha+M$. If we take $x \in U$, then

$$
\left|\left(f+M f^{\prime}\right)(x)\right| \leq 1+M s .
$$

If $x \notin U$, then

$$
\left|\left(f+M f^{\prime}\right)(x)\right| \leq \frac{\alpha}{2}+M .
$$

Hence $\left\|f+M f^{\prime}\right\|<\alpha+M$, but $\alpha+M=(S f)\left(z_{0}\right)+M\left(S f^{\prime}\right)\left(z_{0}\right) \leq\left\|S\left(f+M f^{\prime}\right)\right\|$, which is a contradiction. 
Lemma 3.3 Assume $(f, g) \in A \times B$ and $\left(x_{0}, y_{0}\right) \in \partial A \times \partial B$. If $f\left(x_{0}\right)=g\left(y_{0}\right)=0$, then $T(f, g)(z)=0$ for all $z \in I_{x_{0}, y_{0}}$.

Pro of. Assume, without loss of generality, that $\|f\|=\|g\|=1$ and suppose, contrary to what we claim, that $T(f, g)\left(z_{0}\right)=\alpha \neq 0$ for some $z_{0} \in I_{x_{0}, y_{0}}$.

Let $U:=\left\{x \in X:|f(x)| \geq \frac{\alpha}{2}\right\}$ and $V:=\left\{y \in Y:|g(y)| \geq \frac{\alpha}{2}\right\}$.

As $x_{0}$ is a generalized peak point for $A$, we have $f_{1} \in A$ such that $1=\left\|f_{1}\right\|=\left|f_{1}\left(x_{0}\right)\right|$ and $\left|f_{1}(x)\right|<1$ for all $x \in U$. Similarly, since $y_{0}$ is a generalized peak point for $B$, there exists $g_{1} \in B$ such that $1=\left\|g_{1}\right\|=\left|g_{1}\left(y_{0}\right)\right|$ and $\left|g_{1}(y)\right|<1$ for all $y \in V$. It is apparent that we can assume, multiplying by a constant if necessary, that $T\left(f_{1}, g_{1}\right)\left(z_{0}\right)=1$. Hence, for any positive integers $M$ and $N$, we have

$$
\begin{aligned}
& \left\|T\left(f+M f_{1}, g+N g_{1}\right)\right\| \\
& \quad \geq\left|T\left(f+M f_{1}, g+N g_{1}\right)\left(z_{0}\right)\right| \\
& \quad=\left|T(f, g)\left(z_{0}\right)+N T\left(f, g_{1}\right)\left(z_{0}\right)+M T\left(f_{1}, g\right)\left(z_{0}\right)+M N T\left(f_{1}, g_{1}\right)\left(z_{0}\right)\right| \\
& \quad=\alpha+M N .
\end{aligned}
$$

On the other hand, if $x \in U$,

$$
\left|\left(f+M f_{1}\right)(x)\right| \leq|f(x)|+M\left|f_{1}(x)\right| \leq 1+M s,
$$

where $s<1$ stands for the maximum of $f_{1}$ on $U$. If $x \notin U$,

$$
\left|\left(f+M f_{1}\right)(x)\right| \leq|f(x)|+M\left|f_{1}(x)\right| \leq \frac{\alpha}{2}+M .
$$

Consequently, $\left\|f+M f_{1}\right\| \leq 1+M s$. Similarly, $\left\|g+N g_{1}\right\| \leq 1+N s^{\prime}$. Hence

$$
\begin{aligned}
\alpha+M N & \leq\left\|T\left(f+M f_{1}, g+N g_{1}\right)\right\| \\
& =\left\|f+M f_{1}\right\|\left\|g+N g_{1}\right\| \\
& \leq(1+M s)\left(1+N s^{\prime}\right) \\
& =1+N s+M s^{\prime}+M N s s^{\prime}
\end{aligned}
$$

but it is apparent that we can choose $M$ and $N$ in order to have

$$
1+N s+M s^{\prime}+M N s s^{\prime}<\alpha+M N
$$

which is a contradiction.

Lemma 3.4 If $(x, y)$ and $\left(x^{\prime}, y^{\prime}\right)$ belong to $\partial A \times \partial B$ and are distinct, then $I_{x, y} \cap I_{x^{\prime}, y^{\prime}}=\emptyset$.

Proof. Assume, contrary to what we claim, that there exists $z \in I_{x, y} \cap I_{x^{\prime}, y^{\prime}}$. Let us suppose, without loss of generality, that $x \neq x^{\prime}$.

- If $y \neq y^{\prime}$, then we can choose $f \in C_{x}$ and $g \in C_{y}$ with $f\left(x^{\prime}\right)=g\left(y^{\prime}\right)=0$. Consequently, $|T(f, g)(z)|=1$, but, by Lemma 3.3, $|T(f, g)(z)|=0$, which is a contradiction.

- If $y=y^{\prime}$, then we can choose $f \in C_{x}$ and $g \in C_{y}$ with $f\left(x^{\prime}\right)=0$. Consequently, $|T(f, g)(z)|=1$, but, by Lemma 3.2, $|T(f, g)(z)|=0$, which is a contradiction.

Remark 3.5 The following result can be found in [1]:

Let $A$ be a strongly separating linear subspace of $C_{0}(X)$ and assume that $\partial A \neq \emptyset$. If $S: A \longrightarrow C_{0}(Y)$ is a linear isometry, then there exists a subset of $Y, Y_{0}:=\bigcup_{x \in \partial A} I_{x}$, such that $(S f)(y)=a(y) f(h(y))$ where $h: Y_{0} \longrightarrow \partial A$ is a continuous surjective function and $a(y)=(T g)(y)$ for any $g \in A$ such that $1=\|g\|=g(h(y))$. 
We are now ready to prove our main result:

Theorem 3.6 Let $A$ and $B$ be strongly separating linear subspaces of $C_{0}(X)$ and $C_{0}(Y)$ respectively and assume that $\partial A \neq \emptyset$ and $\partial B \neq \emptyset$. Let $T: A \times B \longrightarrow C_{0}(Z)$ be a bilinear isometry. Then there exist a nonempty $Z_{0}$ of $Z$, a surjective continuous mapping $h: Z_{0} \longrightarrow \partial A \times \partial B$ and a norm-one continuous function $a: Z_{0} \longrightarrow \mathbb{K}$ such that $T(f, g)(z)=a(z) f\left(\pi_{x}(h(z)) g\left(\pi_{y}(h(z))\right.\right.$ for all $z \in Z_{0}$ and every pair $(f, g) \in A \times B$.

Proof. Let us first define a subset $Z_{0}$ of $Z$ as follows:

$$
Z_{0}:=\bigcup_{(x, y) \in \partial A \times \partial B} I_{x, y}
$$

Fix $(x, y) \in \partial A \times \partial B$ and $z \in I_{x, y}$. Let us suppose that $f_{1} \in C_{x}$ and $g_{1} \in C_{y}$. Fix $g^{\prime} \in C_{y}$. Then we can define the following isometries:

$$
\begin{aligned}
& S(f):=T\left(f, g^{\prime}\right), \\
& R(h):=T\left(f_{1}, h\right), \quad \text { where } \quad(f, h) \in A \times B .
\end{aligned}
$$

Then, by Remark 3.5,

$$
\begin{aligned}
T\left(f, g^{\prime}\right)(z) & =(S f)(z) \\
& =S\left(f_{1}\right)(z) f(x) \\
& =T\left(f_{1}, g^{\prime}\right)(z) f(x) \\
& =R\left(g^{\prime}\right)(z) f(x) \\
& =R\left(g_{1}\right)(z) g^{\prime}(y) f(x) \\
& =T\left(f_{1}, g_{1}\right)(z) f(x) g^{\prime}(y) \\
& =a(z) f(x) g^{\prime}(y) .
\end{aligned}
$$

As $\left(f-f(x) f_{1}\right)(x)=0$ and $\left(g-g(y) g_{1}\right)(y)=0$, for any $(f, g) \in A \times B$ we infer, by Lemma 3.3 , that

$$
\begin{aligned}
0 & =T\left(f-f(x) f_{1}, g-g(y) g_{1}\right)(z) \\
& =T(f, g)(z)-f(x) T\left(f_{1}, g\right)(z)-g(y) T\left(f, g_{1}\right)(z)+f(x) g(y) T\left(f_{1}, g_{1}\right)(z) \\
& =T(f, g)(z)-f(x) a(z) f_{1}(x) g(y)-g(y) a(z) f(x) g_{1}(y)+f(x) g(y) a(z) .
\end{aligned}
$$

Hence

$$
T(f, g)(z)=a(z) f(x) g(y)
$$

Let us next define a mapping $h: Z_{0} \longrightarrow \partial A \times \partial B$ as $h(z):=(x, y)$ where $z \in I_{x, y}$. We claim that $h$ is continuous. To this end, fix $z_{0} \in Z_{0}$ and let $h\left(z_{0}\right)=\left(x_{0}, y_{0}\right)$. Let $U$ be a neighborhood of $x_{0}$ and choose $f \in A$ such that $1=\|f\|=\left|f\left(x_{0}\right)\right|$ and $|f|<1$ off $U$. Let $s\left(x_{0}\right)=\sup _{x \in X \backslash U}|f(x)|=\sup _{x \in X \cup\{\infty\} \backslash U}|f(x)|$. It is apparent that $s\left(x_{0}\right)<1$. Similarly, let $V$ be a neighborhood of $y_{0}$ and choose $g \in B$ such that $1=\|g\|=\left|g\left(y_{0}\right)\right|$ and $|g|<1$ off $V$. Let $s\left(y_{0}\right)=\sup _{y \in Y \backslash U}|g(y)|=\sup _{y \in Y \cup\{\infty\} \backslash U}|g(y)|$. As above, $s\left(y_{0}\right)<1$.

Since $h\left(z_{0}\right)=\left(x_{0}, y_{0}\right)$, then $\left|T(f, g)\left(z_{0}\right)\right|=\|T(f, g)\|=1$. Let $s:=\max \left\{s\left(x_{0}\right), s\left(y_{0}\right)\right\}$ and define the following open neighborhood of $z_{0}$ :

$$
W:=\left\{z \in Z_{0}:\left|T(f, g)\left(z_{0}\right)\right|>s\right\} .
$$

Fix $z \in W$ and suppose that $h(z):=(x, y)$. Then, by the above weighted composition representation of $T$,

$$
s<|T(f, g)(z)|=|f(x)||g(y)|,
$$

and, consequently, $|f(x)|>s \geq s\left(x_{0}\right)$ and $|g(y)|>s \geq s\left(y_{0}\right)$. This yields $x \in U$ and $y \in V$, which is to say that $h(W) \subseteq U \times V$ and the proof is done. 
Remark 3.7 The set of generalized peak points, $\partial A$, for a separating linear subspace, $A$, of $C_{0}(X)$ could be empty (see, e.g., [4] or [3]). However, this is not the case for a wide range of subspaces of $C_{0}(X)$ including, for example, extremely regular, or more generally, complete regular subspaces of $C_{0}(X)$ and, above all, function algebras.

Corollary 3.8 Let $A$ and $B$ be completely regular subspaces of $C_{0}(X)$ and $C_{0}(Y)$ respectively. Let $T$ : $A \times B \longrightarrow C_{0}(Z)$ be a bilinear isometry. Then there exist a nonempty $Z_{0}$ of $Z$, a surjective continuous mapping $h: Z_{0} \longrightarrow X \times Y$ and a norm-one continuous function $a: Z_{0} \longrightarrow \mathbb{K}$ such that $T(f, g)(z)=$ $a(z) f\left(\pi_{x}(h(z)) g\left(\pi_{y}(h(z))\right.\right.$ for all $z \in Z_{0}$ and every pair $(f, g) \in A \times B$.

Proof. It is a straightforward consequence of Theorem 3.6 since the set of generalized peak points of a completely regular subspace of $C_{0}(X)$ coincides with $X([2])$.

Corollary 3.9 Let $A$ and $B$ be closed separating subalgebras of $C_{0}(X)$ and $C_{0}(Y)$ respectively, which is to say, non-unital function algebras. Let $T: A \times B \longrightarrow C_{0}(Z)$ be a bilinear isometry. Then there exist a nonempty $Z_{0}$ of $Z$, a surjective continuous mapping $h: Z_{0} \longrightarrow C h(A) \times C h(B)$ and a norm-one continuous function $a: Z_{0} \longrightarrow \mathbb{K}$ such that $T(f, g)(z)=a(z) f\left(\pi_{x}(h(z)) g\left(\pi_{y}(h(z))\right.\right.$ for all $z \in Z_{0}$ and every pair $(f, g) \in A \times B$.

Proof. By Theorem 6.1 in [1], we know that $A$ is a strongly separating subspace of $C_{0}(X)$. Furthermore, by Theorem 2.1 in [7], $\partial A$ coincides with the Choquet boundary for $A$, which is to say that $\partial A$ is a nonempty boundary for $A$. Hence the proof of this corollary is again a straightforward consequence of Theorem 3.6.

Acknowledgements Research partially supported by Spanish Ministery of Science and Technology (MTM2008-04599) and Bancaixa (P1-1B2008-26).

\section{References}

[1] J. Araujo and J. J. Font, Linear isometries between subspaces of continuous functions, Trans. Amer. Math. Soc. 349, No. 1, 413-428 (1997).

[2] B. Cengiz, On extremely regular function spaces, Pacific J. Math. 49, 335-338 (1973).

[3] T. Cho, On the Choquet boundary for nonclosed subspaces of $C(S)$, Pacific J. Math. 35, 575-580 (1970).

[4] H. G. Dales, Boundaries and peak points for Banach function algebras, Proc. London Math. Soc. 22, 121-136 (1971).

[5] H. Holsztyński, Continuous mappings induced by isometries of spaces of continuous functions, Studia Math. 26, 133-136 (1966).

[6] A. Moreno and A. Rodríguez, A bilinear version of Holsztyński’s theorem on isometries of $C(X)$-spaces, Studia Math. 166, 83-91 (2005).

[7] N. V. Rao and A. K. Roy, Multiplicatively spectrum-preserving maps of function algebras. II, Proc. Edinb. Math. Soc. (2) 48, 219-229 (2005). 